\title{
Research and Application of Anti-Collapse Lubricant Compound Polyalcohol for Water-Based Drilling Fluid
}

\author{
Zhang Chujun, Luo Chunzhi, Ye Liyuan, Zhang Huan, Xiang Huan \\ College of Chemistry and Environmental Engineering, Yangtze University, Jingzhou, China \\ Email: 643441175@qq.com
}

How to cite this paper: Zhang, C.J., Luo, C.Z., Ye, L.Y., Zhang, H. and Xiang, H. (2022) Research and Application of Anti-Collapse Lubricant Compound Polyalcohol for WaterBased Drilling Fluid. Open Journal of Yangtze Gas and Oil, 7, 1-12.

https://doi.org/10.4236/ojogas.2022.71001

Received: October 15, 2021

Accepted: December 12, 2021

Published: December 15, 2021

Copyright $\odot 2022$ by author(s) and Scientific Research Publishing Inc. This work is licensed under the Creative Commons Attribution International License (CC BY 4.0).

http://creativecommons.org/licenses/by/4.0/

\begin{abstract}
Polyalcohol is an environmentally friendly anti-collapse lubricant commonly used in water-based drilling fluids. It is generally composed of the single polyether or polyol, but its lubricity and anti-collapse inhibition can't satisfy the application of current water-based drilling fluid systems well. Consequently, the laboratory has carried out research on the anti-collapse lubricant compound polyalcohol. The anti-collapse lubricant samples are mixed with lauryl polyether, nonylphenol polyether and Tween 80 according to different proportions. The cloud point, the reduction rate of lubrication coefficient and linear expansion are measured, and the change they caused should be observed. Then, the optimal ratio is optimized through orthogonal experiments. The results show that the increase in the content of the three kinds of polyethers respectively enhances the inhibition. When the content of lauryl polyether increases, the cloud point of the anti-collapse lubricant declines and the reduction rate of lubrication coefficient rises; when the content of nonylphenol polyether increases, the cloud point declines, but it has little effect on the reduction rate of the lubrication coefficient; when the content of Tween 80 increases, the cloud point rises, and the reduction rate of the lubrication coefficient first augments and then basically remains unchanged. The best mass ratio of lauryl polyether:TW-80:nonylphenol polyether is 6:3:4.
\end{abstract}

\section{Keywords}

Polyalcohol, Lubricity, Inhibition, Lauryl Polyether, Nonylphenol Polyether, Tween 80, Cloud Point

\section{Introduction}

Polyalcohol [1] is an environmentally friendly anti-collapse lubricant for water- 
based drilling fluids that emerged in the 1980s and 1990s which is generally composed of the single polyether or polyol. The polyether used initially is a random copolymer of Ethylene Oxide (EO) and Propylene Oxide (PO) with ethylene glycol as an initiator, which has a cloud point effect and provides certain lubricity, inhibition and blocking effect, etc. Polyol type polyalcohol [2] [3], mostly polyethylene glycol, polyvinyl alcohol, etc., which generally has no Cloud Point (CP) but good inhibition, is easy to form intermolecular crosslinks and to chelate with formation water or metal ions in drilling fluids. It has a great impact on drilling fluid function but soon be eliminated. By contrast, the application of polyether polyalcohol has been promoted. The increasing difficulty of drilling calls for higher requirements for water-based anti-collapse polyether lubricants, especially the requirements for lubricity. Traditional polyalcohol requires the reduction rate of lubrication coefficient greater than $50 \%$ and the reduction rate of linear expansion greater than $15 \%$. However, the current standard is that the former is greater than $70 \%$ and the latter is greater than $20 \%$. As a result, it is an inevitable trend to study the anti-collapse lubricant compound polyalcohol. This experiment explored the influence of concentration of lauryl polyether, nonylphenol polyether and Tween 80 on the $\mathrm{CP}$, the reduction rate of lubrication coefficient and linear expansion, and then the changes of them after mixing are explored. Based on the above procedures, a number of anti-collapse lubricant samples were prepared by the orthogonal experiment method. Then, the excellent anti-collapse lubricant compound polyalcohol is selected, which has been used in Changqing Oilfield and achieved good results.

\section{Experimental Materials and Instruments}

\subsection{Experimental Materials}

lauryl polyether (JHCJ-1, industrial reagent, 99\%), nonylphenol polyether (JHCL-3, industrial reagent, 99\%), Tween 80 (TW-80, industrial reagent, 99\%), sodium carbonate (AR) and Bohai drilling bentonite(industrial reagent, 99\%), etc.

\subsection{Experimental Instruments}

beaker, glass rod, electronic balance (ST-02, Maximum is $300.00 \mathrm{~g}$ ), alcohol thermometer $\left(0-100^{\circ} \mathrm{C}\right)$, super constant temperature water bath (HH-501/601S, Jiangsu Jincheng Guosheng Experimental Instrument Factory), frequency conversion high-speed stirrer (GJS-B12K, Qingdao Haitongda Special Instrument Factory), cups for high stirring $(800 \mathrm{ml})$, tablet press machine (XCX-16T, Qingdao Haitongda Special Instrument Factory), extreme pressure lubrication instrument (Fann212, Fann Instrument Company), room temperature expansion tester (NP-01, Qingdao Haitongda Special Instrument Factory), multifunctional blast drying oven (101-1, Qingdao Haitongda Special Instrument Factory).

\section{Experimental Methods}

\subsection{Sample Preparation}

Weigh JHCJ-1, JHCL-3 and TW-80 in a beaker according to different mass rati- 
os, and stir evenly.

\subsection{Determination of $\mathrm{CP}$}

Accurately weigh $1 \mathrm{~g}$ sample of anti-collapse lubricant into a beaker, dissolve it with $100 \mathrm{~g}$ distilled water, and stir evenly. Take $5 \mathrm{ml}$ of that into a dry and clean test tube, which then placed into a super constant temperature water bath, the liquid level in the test tube should be lower than that of water bath. Constantly shake the test tube horizontally to make it evenly heated, and measure the temperature of the solution with a thermometer. When the temperature is approaching to the $\mathrm{CP}$, the heating rate should be controlled at $0.1^{\circ} \mathrm{C} / 5 \mathrm{~min}$. When the onset of surprising clouding in the solution was noted, and then solution gets clear again when the temperature drops, the temperature at this time is the CP. The average value of three parallel determinations is the CP of the sample.

\subsection{Determination of the Reduction Rate of Lubrication Coefficient}

1) Preparation of base slurry: weigh 2 parts of $16 \mathrm{~g}$ sodium bentonite, add them respectively to $400 \mathrm{ml}$ distilled water, stir at high speed for $5 \mathrm{~min}$. then add 0.86 g sodium carbonate, continue to stir for $15 \mathrm{~min}$, and maintain at room temperature for 24 hours.

2) Preparation of sample slurry: add 2.0 g sample to a cup of prepared base slurry, and stir at 11,000 r/min for $5 \mathrm{~min}$.

3) Determination of the reduction rate of lubrication coefficient: on the extreme pressure lubrication instrument, determine the lubrication coefficient of base slurry $K_{f 0}$ and the lubrication coefficient $K_{f 1}$ of sample slurry according to the method of use of the instrument. The calculation formula for the reduction rate of the lubrication coefficient is as below:

$$
R=\frac{K_{f 0}-K_{f 1}}{K_{f 0}} \times 100
$$

where $R$ is the reduction rate of the lubrication coefficient (\%), $K_{f 0}$ is the lubrication coefficient of base slurry, $K_{f 1}$ is the lubrication coefficient of sample slurry. In this study, $K_{f 0}$ is calculated as 0.54 . The higher the reduction rate of lubrication coefficient, the better lubricity.

\subsection{Determination of the Reduction Rate of Linear Expansion}

Accurately weigh $10.0 \mathrm{~g}$ calcium bentonite dried to constant weight at $101^{\circ} \mathrm{C} \pm$ $3^{\circ} \mathrm{C}$ in the measuring cylinder, and keep it on the tablet press machine for $5 \mathrm{~min}$ under the pressure of $4 \mathrm{MPa}$ to get the experimental core. Install the measuring cylinder with the core on the dilatometer, pour distilled water or $6 \%$ sample aqueous solution into the measuring cylinder and record the expansion height $\mathrm{H}$ of the core soaked in distilled water or sample solution for 8 hours. The calculation formula for the reduction rate of linear expansion is as below:

$$
B=\frac{\Delta H_{1}-\Delta H_{2}}{\Delta H_{1}} \times 100
$$


where $B$ is the reduction rate of linear expansion (\%), $\Delta H_{1}$ is the core linear expansion height after immersed in distilled water for 8 hours (mm), $\Delta H_{2}$ is the core linear expansion height after immersed in $6 \%$ sample solution for 8 hours (mm). In this study, $\Delta H_{1}$ is calculated as $9.530 \mathrm{~mm}$. The higher the reduction rate of linear expansion, the better inhibition.

\section{Results and Analysis}

\subsection{The Influence of Single Agent Concentration on CP, Lubricity and Inhibition}

Prepare the aqueous solutions of JHCJ-1 and JHCL-3 with different mass fractions, and determine the CP according to the experimental method 3.2. The experimental results are shown in Figure 1. Because the CP of TW-80 is very high, the error of measuring its CP under normal pressure is relatively large, TW-80 will not be discussed here. According to the methods of 3.3 and 3.4, the reduction rate of lubrication coefficient and linear expansion with different single concentrations were measured. The experimental results are illustrated in Figure 2 and Figure 3.

It's known from Figure 1 that the CP of non-ionic surfactants (NS) JHCJ-1 and JHCL-3 both decline first and then rise with the increase of concentration. This phenomenon can be explained by the shape of micelles in aqueous solution changes with concentration [4]. Before the CP reaches the lowest value, the increase of the surfactant concentration only improves the number of micelles, increases the probability of collision and coalescence. Therefore, it is easy to cause phase separation and reduce the $\mathrm{CP}$. When the shape of the micelle changes from a spherical shape to a rod shape, the gyration radius of the micelle particles increases, which improves the viscosity of the solution, consequently, the $\mathrm{CP}$ rises. Figure 2 illustrates that the lubricity of the system increases with the addition of NS. The longitudinal comparison shows that the lubricity of TW-80 is

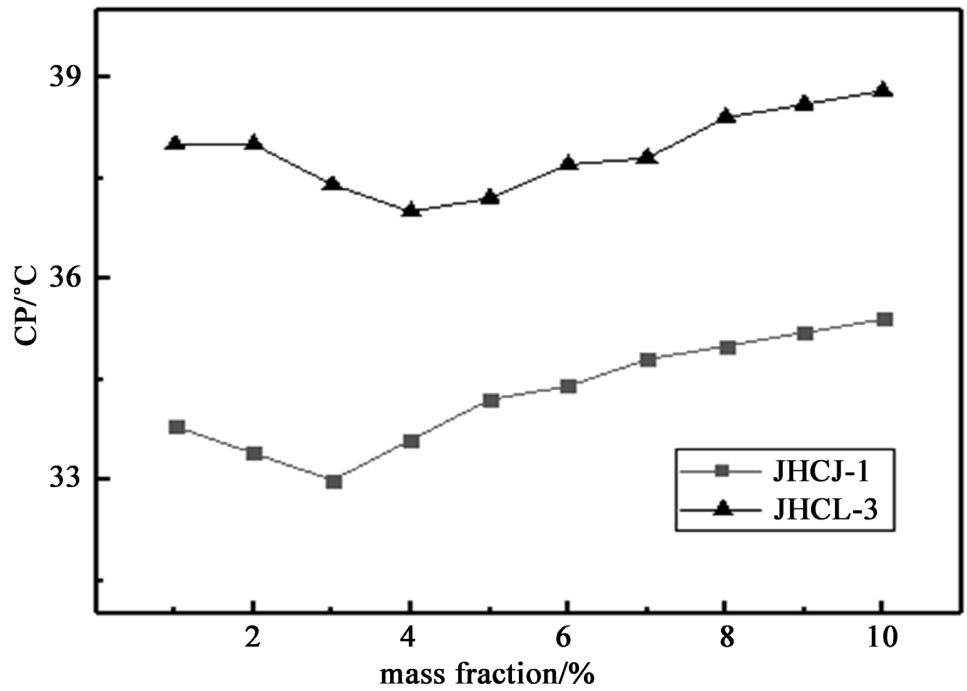

Figure 1. CP change with concentration. 


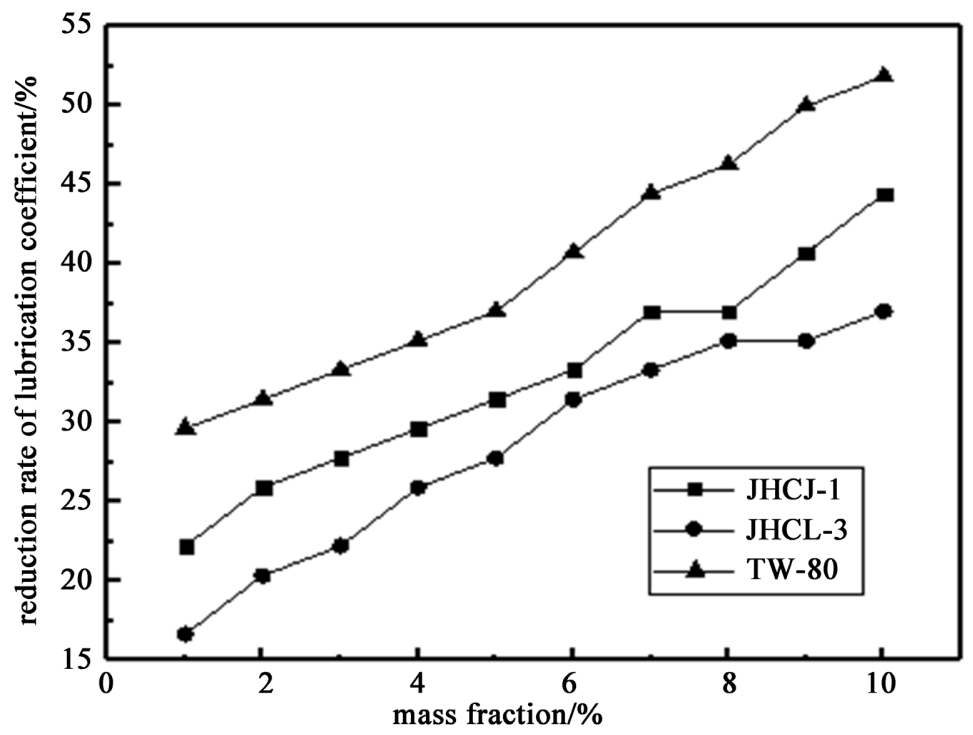

Figure 2. Change of the reduction rate of lubrication coefficient with concentration.

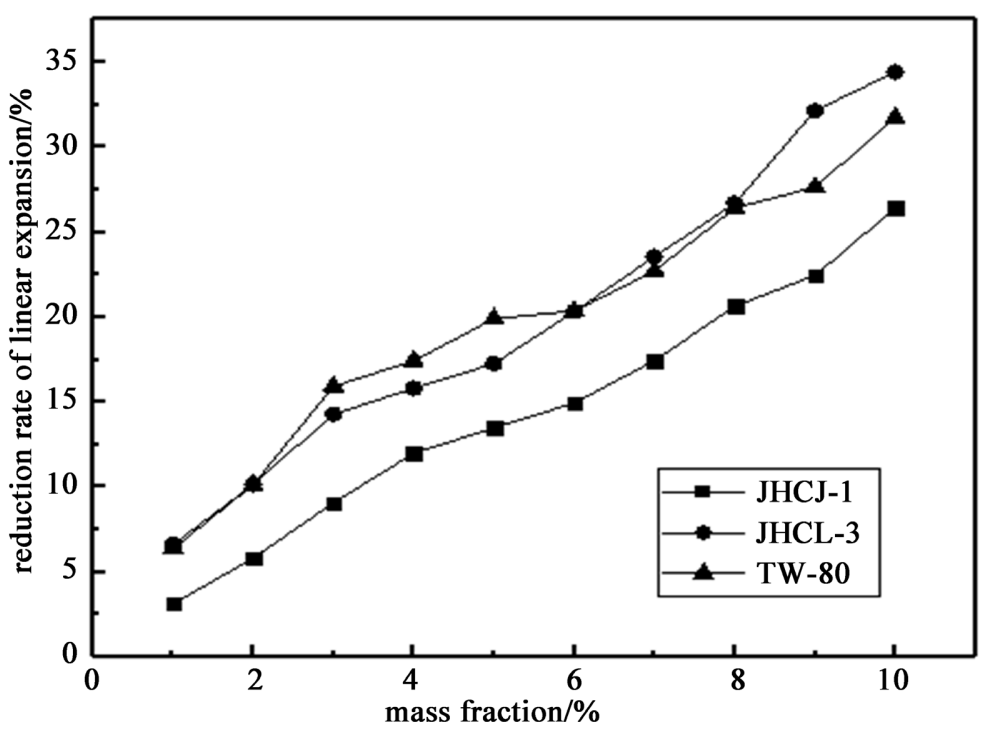

Figure 3. Change of the reduction rate of linear expansion with concentration.

the best, followed by JHCJ-1, which is related to the dramatic difference in hydrophobic structure. Figure 3 shows the same trend of inhibitory effect about the system, with JHCJ-1 having the worst performance, and TW-80 and JHCL-3 being similar.

\subsection{CP, Lubricity and Inhibition of Anti-Collapse Lubricant Compound Polyalcohol}

Mix JHCJ-1, JHCL-3 and TW-80 in accordance with the mass ratio in Table 1, and then determine the $\mathrm{CP}$ of the sample's $1 \%$ aqueous solution, the reduction rate of lubrication coefficient with $0.5 \%$ addition and the reduction rate of linear expansion of $6 \%$ aqueous solution according to the methods 3.2, 3.3 and 3.4., the experimental results are respectively shown in Figures 4-6. 
Table 1. Preparation of anti-collapse lubricant compound polyalcohol.

\begin{tabular}{cccc}
\hline Serial number & $\begin{array}{c}\text { JHCJ-1:JHCL-3:TW-80 } \\
\text { (Mass ratio) }\end{array}$ & Serial number & $\begin{array}{c}\text { JHCJ-1:JHCL-3:TW-80 } \\
\text { (Mass ratio) }\end{array}$ \\
\hline $1-1$ & $1: 1: 1$ & $2-4$ & $1: 4: 1$ \\
$1-2$ & $2: 1: 1$ & $2-5$ & $1: 5: 1$ \\
$1-3$ & $3: 1: 1$ & $2-6$ & $1: 6: 1$ \\
$1-4$ & $4: 1: 1$ & $3-2$ & $1: 1: 2$ \\
$1-5$ & $5: 1: 1$ & $3-3$ & $1: 1: 3$ \\
$1-6$ & $6: 1: 1$ & $3-4$ & $1: 1: 4$ \\
$2-2$ & $1: 2: 1$ & $3-5$ & $1: 1: 5$ \\
$2-3$ & $1: 3: 1$ & $3-6$ & $1: 1: 6$ \\
\hline
\end{tabular}

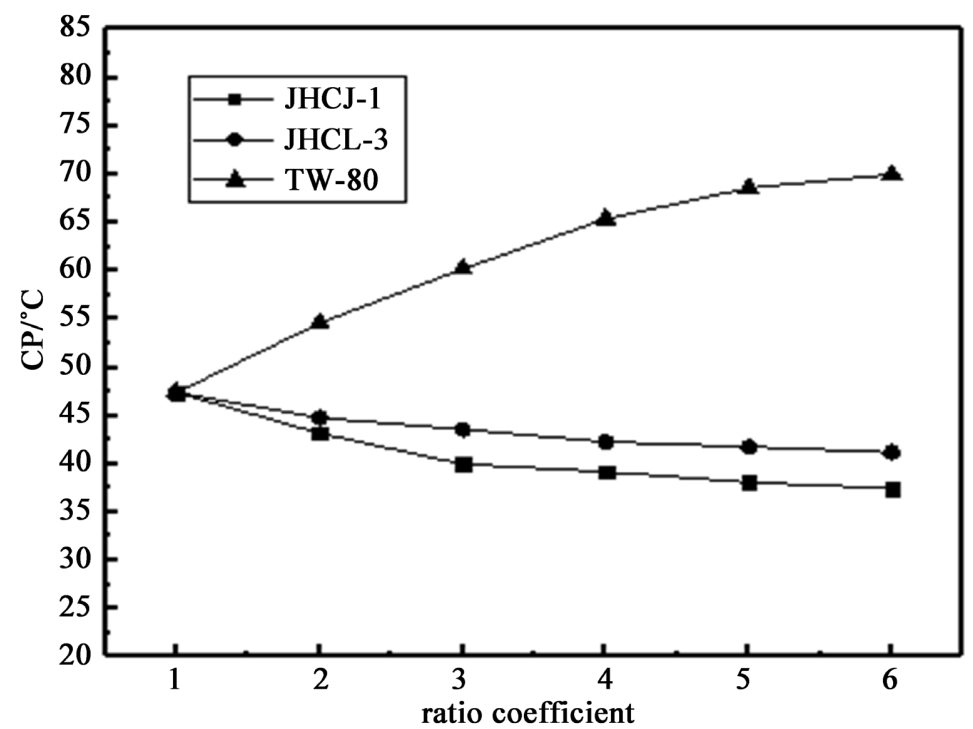

Figure 4. CP change with ratio.

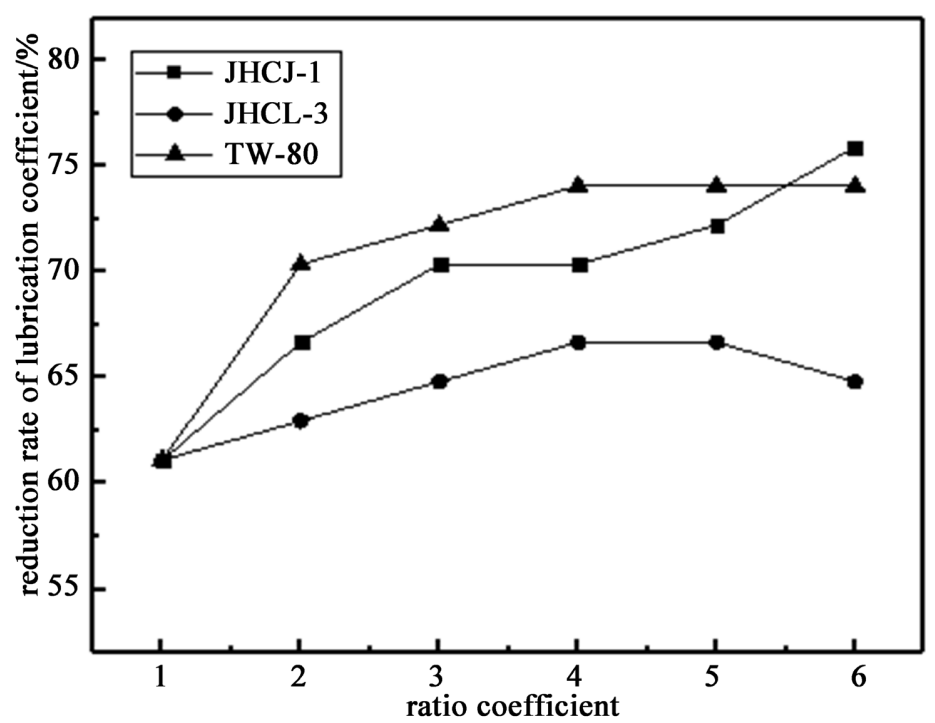

Figure 5. Change of the reduction rate of lubrication coefficient with ratio. 


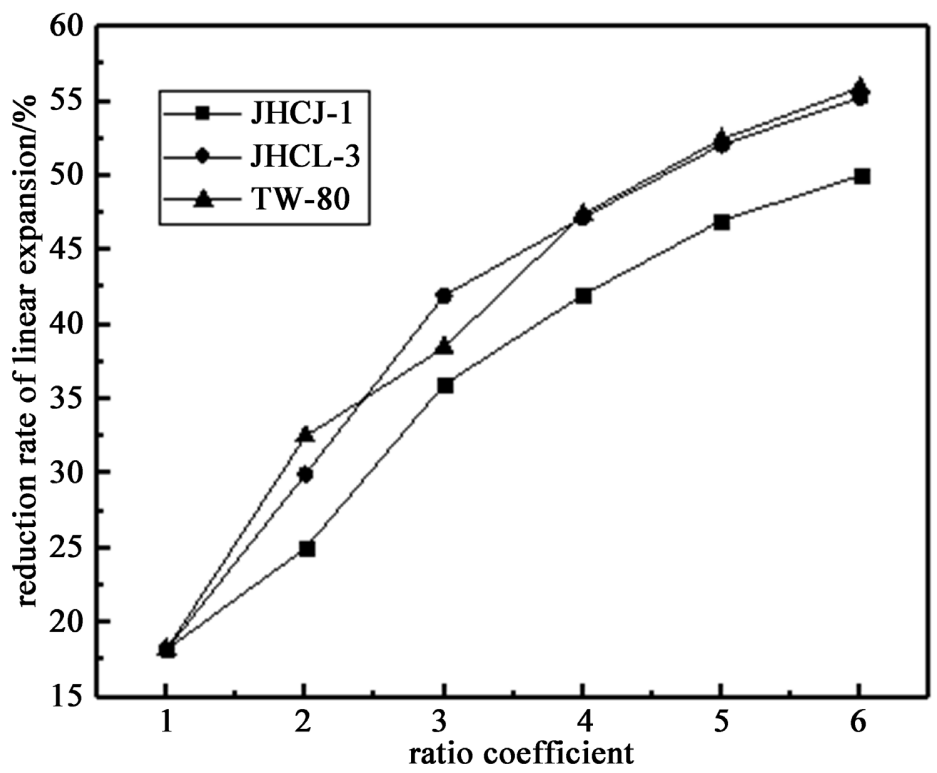

Figure 6. Change of the reduction rate of linear expansion with ratio.

Figure 4 shows that when the proportions of other components remain unchanged, the CP of mixed system declines with the increase proportion of JHCJ-1 or JHCL-3, and rises with the increase proportion of TW-80. It is due to the former is solubilized in the micellar barrier layer, causing the micelle volume to expand, thereby reducing the CP of mixed NS. By contrast, the latter reduces the polarity of the medium by changing the structure of the solvent water. At the same time, part of the organic matter is adsorbed on the micelle-water interface. Through its solvation, the micellization of NS is limited, resulting in an increase in the CP [5]. In addition, the length of the hydrophobic alkyl chain is also effective. For homologs, the longer the carbon chain of organic, the lower the concentration of organic required for phase separation of the NS aqueous solution. Therefore, the relative solubility of NS and other organics in water determines the change in CP [6].

Figure 5 demonstrates that when the proportions of other components remain unchanged, the lubricity of mixed system rises with the increase of the amount of JHCJ-1, meanwhile, with the increase of the amount of TW-80, the lubricity of mixed system first rises sharply and then gets stable. JHCL-3 has little effect on lubricity. The adsorption model of lubricant on the surface of friction pair is shown in Figure 7 [7]. The lubricating effect of the lubricant is closely related to its adsorption group, the strength of boundary film, and the hydrophobicity of oil film. JHCJ-1, TW-80, and JHCL-3 are all alkyl alcohol polyether lubricants, and the adsorption groups are ether bonds and hydroxyl groups, so the lubricating ability mainly depends on the number of hydrophobic alkyl chains and adsorption groups. For the homologous organics, the increase in the chain length of oily molecules is conducive to the lubrication of the metal surface. When the number of carbon atoms in the chain improves to $16-18$, the influence of the chain length basically disappears. After that, even if the carbon chain grows, 


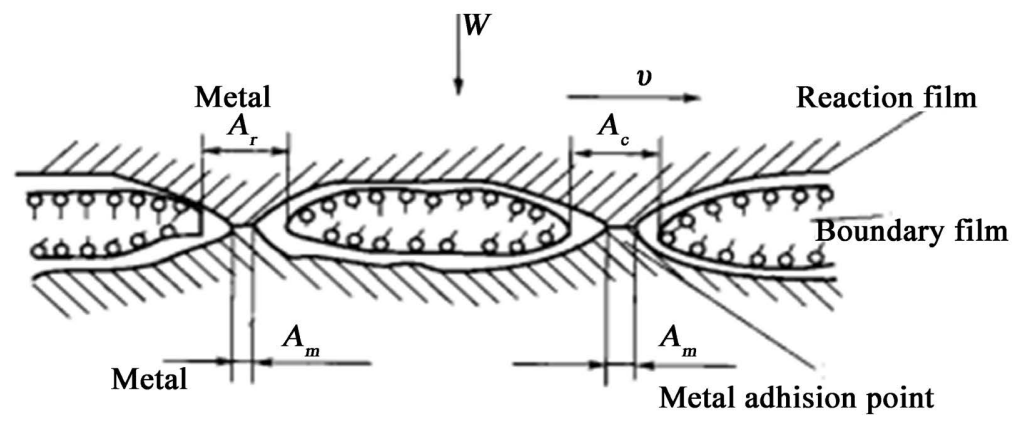

Figure 7. Adsorption model.

the lubricating ability of organics will be reduced. With the increase of carbon atoms, the HOMO energy level rises, the LUMO energy level declines, the difference between two energy levels gradually decreases, the interaction force between molecules increases, the molecules will be more closely arranged, and the cohesion will also be enhanced. So, the bearing capacity of the surface adsorption film becomes stronger, which is more conducive to reducing friction [8]. The rigid group in the hydrophobic chain of JHCL-3 contributes little to the lubricating ability of the molecule. Therefore, the hydrophobic chain in JHCL-3 can be regarded as containing only 9 carbon atoms, that is to say the lubricity of JHCJ-1 is better than that of JHCL-3. TW-80 and JHCJ-1 are not completely homologous, but the long chain structure, 18 carbon atoms, and high CP phenomenon of TW-80 indicates that TW-80 has strong hydrophobic chain and high polarity, so TW-80 is lubricated better than JHCJ-1. At the same time, due to the long hydrophobic chain of TW-80, it is not easy to enter the gap of the extreme pressure friction surfaces. As a result, the excellent lubricating performance of TW-80 cannot be shown with the increase in dosage, and the strong foaming ability of TW-80 has a negative effect.

Figure 6 indicates that when the proportions of other components remain unchanged, the inhibition of mixed system rises with the increase of NS addition, which is consistent with the previous results.

\subsection{Optimum of Anti-Collapse Lubricant Compound Polyalcohol}

Through the above experiments, we have recognized the respective effects of three non-ionic surfactants on the $\mathrm{CP}$, lubricity and inhibition of the mixed system. The composite polyalcohol sample was prepared by orthogonal experiment method, the $\mathrm{CP}$ of the sample's $1 \%$ aqueous solution, the reduction rate of lubrication coefficient with $0.5 \%$ addition and linear expansion of $6 \%$ aqueous solution were measured, and results are shown in Table 2 . Summary and analysis for the primary and secondary factors affecting performance to find the best mixing ratio to determine the best formula are done.

The choice of the $\mathrm{CP}$ range, when applying polyalcohol in the field, is related to the down-hole temperature, and the suitable $\mathrm{CP}$ temperature is generally between $30^{\circ} \mathrm{C}$ and $70^{\circ} \mathrm{C}$. Therefore, the assessment indicators in this orthogonal experiment mainly consider the reduction rate of lubrication coefficient and linear 
expansion, $C P$ just for reference. From $K_{i j}$, it can be concluded that $A_{2} B_{2} C_{3}$, $\mathrm{A}_{2} \mathrm{~B}_{3} \mathrm{C}_{3}, \mathrm{~A}_{3} \mathrm{~B}_{2} \mathrm{C}_{3}$ and $\mathrm{A}_{3} \mathrm{~B}_{3} \mathrm{C}_{3}$ are good, that is, the mass ratios of JHCJ-1, TW-80 and JHCL-3 are 5:2:4, 5:3:4, 6:2:4 and 6:3:4.

According to the optimal formula of the orthogonal experiment, the anti-collapse lubricant composite polyalcohol was measured and results are shown in Table 3.

The technical requirements of the lubricating polyalcohol treatment agent for drilling fluids in Changqing Oilfield requires a $\mathrm{CP}$ is $30^{\circ} \mathrm{C}-50^{\circ} \mathrm{C}$, a reduction rate of lubrication coefficient greater than $70 \%$, and a reduction rate of linear expansion greater than $20 \%$. The data of the above optimal formula fully meets

Table 2. Orthogonal experiment scheme and result analysis.

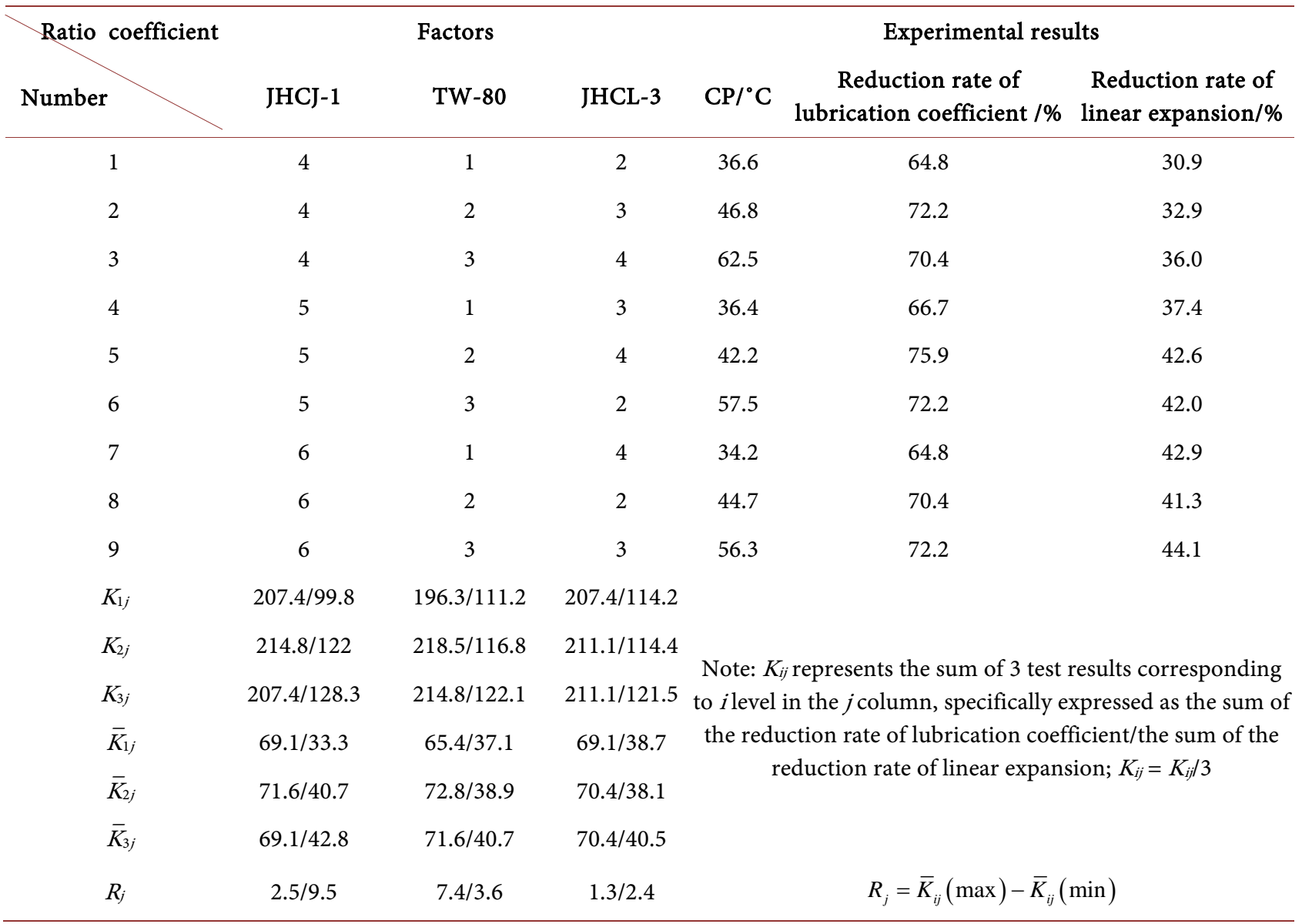

Table 3. The performance of optimal formula anti-collapse lubricant composite polyalcohol.

\begin{tabular}{cccc}
\hline Formula & $\mathrm{CP} /{ }^{\circ} \mathrm{C}$ & $\begin{array}{c}\text { Reduction rate of lubrication } \\
\text { coefficient/\% }\end{array}$ & $\begin{array}{c}\text { Reduction rate of linear } \\
\text { expansion/\% }\end{array}$ \\
\hline $\mathrm{A}_{2} \mathrm{~B}_{2} \mathrm{C}_{3}$ & 43.4 & 74.1 & 45.7 \\
$\mathrm{~A}_{2} \mathrm{~B}_{3} \mathrm{C}_{3}$ & 45.2 & 76.9 & 47.6 \\
$\mathrm{~A}_{3} \mathrm{~B}_{2} \mathrm{C}_{3}$ & 41.6 & 75.2 & 45.9 \\
$\mathrm{~A}_{3} \mathrm{~B}_{3} \mathrm{C}_{3}$ & 44.4 & 78.5 & 48.2 \\
\hline
\end{tabular}


the requirements of Changqing Oilfield, among which $\mathrm{A}_{3} \mathrm{~B}_{3} \mathrm{C}_{3}$ formula is the best.

\subsection{The Mechanism of Anti-Collapse Lubricant Compound Polyalcohol}

Through the above research, it is obvious that the lubricity and inhibition of the anti-collapse lubricant compound polyalcohol is definitely better than that of single traditional polyether. Compared with the latter, the former has a $20 \%-30 \%$ increase in the reduction rate of lubrication coefficient and a 30\% - 35\% increase in the reduction rate of linear expansion, which is doubled. The wide use of polyalcohol benefits from the ability to take into account the lubricity and inhibition of water-based drilling fluids, which are not available in other water-based lubricants. The lubricity and inhibition of polyalcohol are inevitably associate with the hydrophobic chain structure of NS, the proportion of EO and PO, molecular weight and cloud point effect are inevitable [9]. The hydrophilic group is adsorbed on the clay particles or the friction surface, and the hydrophobic group is stretched outward to form a strong oil film. There are synergistic and intermolecular effects among the three polyethers, and these kinds of effect increase the power of the hydrophobic oil film, simultaneously, the intermolecular interaction allows more TW-80 to enter the narrow working range, which makes the oil film more lipophilic, thus showing better performance.

\section{Application of Anti-Collapse Lubricant Compound Polyalcohol}

According to the $\mathrm{A}_{3} \mathrm{~B}_{3} \mathrm{C}_{3}$ formula, Jingzhou Xuecheng Industrial Co., Ltd. uses industrial materials and water to produce anti-collapse lubricant compound polyalcohol, and the product used in Changqing Oilfield in March 2021 and September 2021 reaches 2000 tons. When the addition is 1\% in drilling fluid of Changqing Oilfield, the drilling fluid torque decreases from $42 \mathrm{kN} \cdot \mathrm{m}-46 \mathrm{kN} \cdot \mathrm{m}$ to 15 $\mathrm{kN} \cdot \mathrm{m}$, the rheology is basically unchanged, the mud cake is thin and tough, the cuttings particles are large, and the edges and corners are clear. The product was tested in Tianjin Petroleum Industry Oil Well Cement and Admixtures Quality Supervision and Testing Center, the results and conclusion are respectively shown in Figure 8 and Figure 9. All the indicators meet the requirements of Jingzhou Xuecheng Q/JZXC 008-2019.

\section{Conclusions}

1) The CP temperature of JHCJ-1 and JHCL-3 declines first and then rises as the concentration increases. This discovery can provide a range of concentration options for the field application of the cloud point effect.

2) The lubricity and inhibition of the single-agent rise with the increase of the concentration. The lubricity of TW- 80 is the best when the mass fraction is the same; the inhibition of TW- 80 is best when the mass fraction is less than $6 \%$, 
钻井液用防塌润滑剂 复合聚合醇 XCS-III 检验结果

Result of anti-collapse lubricant fluid compound polyalcohol XCS-III

\begin{tabular}{|c|c|c|c|c|}
\hline $\begin{array}{c}\text { 检验项目 } \\
\text { Inspection index }\end{array}$ & $\begin{array}{c}\text { 标准规定指标 } \\
\text { Standard index }\end{array}$ & $\begin{array}{c}\text { 检验结果 } \\
\text { Result }\end{array}$ & $\begin{array}{c}\text { 单项判定 } \\
\text { Judgment }\end{array}$ & $\begin{array}{c}\text { 检验方法 } \\
\text { Method }\end{array}$ \\
\hline $\begin{array}{c}\text { 外观 } \\
\text { Appearance } \\
\text { 无色、微乳白色或 } \\
\text { Colorless, slightly } \\
\text { milky or yellow } \\
\text { viscous liquid }\end{array}$ & $\begin{array}{c}\text { 黄色粘暞液体 } \\
\text { Yellow viscous liquid }\end{array}$ & $\begin{array}{c}\text { 合格 } \\
\text { Qualified }\end{array}$ & $\begin{array}{c}\text { Q/JZXC } \\
008\end{array}$ \\
\hline $\begin{array}{c}\text { 浊点, }{ }^{\circ} \text { C } \\
\text { Cloud point }\end{array}$ & $30 \sim 50$ & 37 & $\begin{array}{c}\text { 合格 } \\
\text { Qualified }\end{array}$ & $\begin{array}{c}\text { Q/JZXC } \\
008\end{array}$ \\
\hline $\begin{array}{c}\text { 注滑系数降低率, \% } \\
\text { Reduction rate of } \\
\text { lubrication coefficient }\end{array}$ & $\geqslant 70$ & 79 & $\begin{array}{c}\text { 合格 } \\
\text { Qualified }\end{array}$ & $\begin{array}{c}\text { Q/JZXC } \\
008\end{array}$ \\
\hline $\begin{array}{c}\text { 起泡率, \% } \\
\text { Foaming rate }\end{array}$ & $\leqslant 8$ & 7 & $\begin{array}{c}\text { 合格 } \\
\text { Qualified }\end{array}$ & $\begin{array}{c}\text { Q/JZXC } \\
008\end{array}$ \\
\hline $\begin{array}{c}\text { 线性膨胀降低率, \% } \\
\text { Reduction rate of } \\
\text { linear expansion }\end{array}$ & $\geqslant 20$ & 44 & $\begin{array}{c}\text { 合格 } \\
\text { Qualified }\end{array}$ & $\begin{array}{c}\text { Q/JZXC } \\
008\end{array}$ \\
\hline
\end{tabular}

Figure 8. Product inspection results.

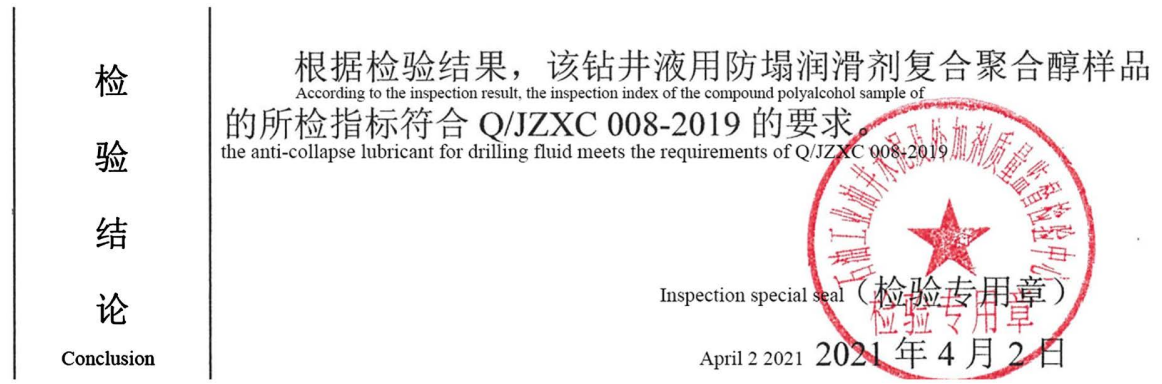

Figure 9. Product inspection conclusion.

and the inhibition of JHCL-3 is best when the mass fraction is more than $6 \%$. This discovery can provide research directions for finding more excellent lubricants and inhibitors.

3) In the ternary mixed system, when the ratio of the other two non-ionic surfactants is unchanged, the increase of JHCJ-1 or JHCL-3 will reduce the CP of the mixed system, and the increase of TW- 80 makes the $\mathrm{CP}$ of the mixed system rises. The increased amount of JHCJ-1 improves the lubricity of the mixed system, JHCL-3 has a small effect on the lubricity of the mixed system, and the increased amount of TW- 80 improves the lubricity at first and then basically remains unchanged. Finally, the addition of the three kinds of NS was increased, causing the improvement of inhibition of the mixed system.

4) According to the optimal formula selected by the orthogonal experiment, the performance of the polyalcohol product formulated with $\mathrm{A}_{3} \mathrm{~B}_{3} \mathrm{C}_{3}$ fully meets the technical requirements of the lubricating polyalcohol treatment agent for drilling fluids in Changqing Oilfield and the standard requirements of Q/JZXC 008-2019 of Jingzhou Xuecheng Industrial, Co., Ltd. During field application, the drilling fluid 
torque is significantly reduced, the rheology is not affected, the mud cake is thin and tough, and the drilling cuttings have sharp edges and corners.

\section{Acknowledgments}

First of all, I would like to express my sincere gratitude to my teacher, Professor Luo Chunzhi, who guided me in the design of the experiment and the writing of the thesis. Without her enlightening guidance and patience, this paper would not have reached its current form. I also genuinely thank my classmates Zou Yanlin, Wang Baijing, Zhang Huan and Xiang Huan in the laboratory for their support and encouragement.

\section{Conflicts of Interest}

The authors declare no conflicts of interest regarding the publication of this paper.

\section{References}

[1] Qin, G.C., Xu, M.B., He, M., et al. (2021) Research Progress in the Application of Polyalcohol in Drilling Fluids. Polymer Bulletin, 65-72.

[2] Song, B.T., Liu, Z.L. and Xue, Y. (2014) Application of Polyether Polyol Drilling Fluid in $\mathrm{Xu} 33$ Block of Subei Basin. Drilling Fluids and Completion Fluids, 31, 92-94.

[3] Gao, Y.H., Liu, X.D. and Tan, W.L. (2014) Research on Additives to Improve the Performance of Polyether Polyol Drilling Fluids. Drilling Fluids and Completion Fluids, 31, 5-8.

[4] Qiao, L. and Easteal, A.J. (1998) The Interaction between Triton X Series Surfactants and Poly (Ethylene Glycol) in Aqueous Solutions. Colloid \& Polymer Science, 276, 313-320. https://doi.org/10.1007/s003960050245

[5] Wu, H.Y., Zhang, D.C. and Yu, X.D. (2019) Determination of the Cloud Point of Tween 80 and Its Influencing Factors. Food Industry, 40, 184-186.

[6] Jiang, X.F., Wang, M.M. and Li, Y. (2015) Preparation of a Novel Triazine Ring Nonionic Surfactant. Journal of Dalian University of Technology, 34, 353-356.

[7] Li, G.R. and Wang, C.J. (2020) Research Progress and Development Trend of Polar Adsorption Drilling Fluid Lubricants. Drilling Fluids and Completion Fluids, 37, 9-12.

[8] Wang, H.Q. (2018) Research on Competitive Adsorption and Lubrication Performance of Biodiesel Components Based on Molecular Simulation. Master's Thesis, Jiangsu University, Zhenjiang.

[9] Geng, Y., Liu, H.S. and He, Z.T. (2021) Preparation of a New Environmentally Friendly Lubricant and Its Application in Shale Gas Water-Based Drilling Fluids. Drilling and Production Technology, 44, 92-95. 